\title{
Controllability of neutral impulsive stochastic functional in- tegrodifferential equations driven by a fractional Brownian motion with infinite delay via resolvent operator
}

\author{
Dimplekumar Chalishajar ${ }^{a, *}$, K. Ramkumar ${ }^{b}$, A. Anguraj ${ }^{b}$, K. Ravikumar $^{\mathrm{b}}$, Mamadou Abdoul Diop $^{\mathrm{c}}$ \\ ${ }^{a}$ Department of Mathematics and Computer science, Mallory Hall, Virginia Military Institute, Lexington, VA 24450, USA. \\ ${ }^{b}$ Department of Mathematics, PSG College of Arts and Science, Coimbatore, 641 046, India. \\ ${ }^{c}$ Departement de Mathematiques, Universite Geston Berger de Sanit-Louis, UFR SAT-St Louis, Senegal.
}

\begin{abstract}
This paper is concerned with the controllability results of neutral impulsive stochastic functional integrodifferential equations driven by a fractional Brownian motion with infinite delay in a real separable Hilbert space. The controllability results are obtained using stochastic analysis, the theory of resolvent operator in the sense of Grimmer and Krasnoselskii fixed point theorem. An example is provided to illustrate the obtained theory.
\end{abstract}

Keywords: Controllability, impulsive systems, fractional Brownian motion, neutral functional integrodifferential equations, resolvent operator, infinite delay.

2020 MSC: 35R10, 60G22, 60H20, 93B05.

(C)2022 All rights reserved.

\section{Introduction}

The concept of controllability plays a major role in both finite and infinite dimensional spaces for systems represented by ordinary differential equations and partial differential equations. One of the basic qualitative behaviors of a dynamical system is the controllability. The problem of controllability is to show the existence of control function, which steers the solution of the system from its initial state to final state, where the initial and final states may very over the entire space. Conceived by Kalman, the controllability concept has been studied extensively in the fields of finite and infinite-dimensional systems. If a system cannot be controlled completely then different types of controllability can be defined such as approximate, null, local null and local approximate null controllability. For more details the reader may refer to $[1,9,10,17,18]$ and references therein.

On the other hand, the properties of long/short-range dependence are widely used in describing many phenomena in fields like hydrology and geophysics as well as economics and telecommunications. As

\footnotetext{
*Corresponding author

Email addresses: chalishajardn@vmi.edu (Dimplekumar Chalishajar), ramkumarkpsg@gmail.com (K. Ramkumar), angurajpsg@yahoo.com (A. Anguraj), ramkumarkpsg@gmail.com (K. Ravikumar), dipu17370@gmail.com (Mamadou Abdoul Diop)
}

doi: $10.22436 /$ jnsa.015.03.01

Received: 2021-10-01 Revised: 2021-11-01 Accepted: 2021-11-19 
extension of Brownian motion, fractional Brownian motion is a self-similar Gaussian process which has the properties of long/short-range dependence. However, fractional Brownian motion is neither a semi martingale nor a Markov process. In [4, 5, 11, 12], studied the general theory for the infinite-dimensional stochastic differential equations driven by a fractional Brownian motion.

Few years ago, Park et al. [15] investigated the controllability of impulsive neutral integrodifferential systems with infinite delay in Banach spaces using Schauder-fixed point theorem. Very recently, $[2,7]$ established the existence, uniqueness and asymptotic behaviors of mild solutions to a class of impulsive neutral stochastic integrodifferential equations driven by a fractional Brownian motion with delays. Moreover, several upcoming researchers are keen interested to study the solution of control problems in the field of stochastic systems. A through survey of literature reveals that a very little work has been done for the fractional Brownian motion in stochastic control problems. Chen [6] concerned the approximate controllability for semilinear stochastic equations with fractional Brownian motion. Several researchers reported the use of fractional Brownian motion in stochastic integrodifferential equations (see $[11,12,17]$ and references therein). Moreover, the controllability of neutral impulsive stochastic functional integrodifferential systems with infinite delay driven by a fractional Brownian motion is an untreated topic in the literature so far. Thus, we will make the first attempt to study such problem in this paper.

The goal of present research work is focus to study the controllability of neutral impulsive stochastic functional integrodifferential equations of the form:

$$
\begin{aligned}
d\left[x(t)-g\left(t, x_{t}\right)\right]= & A\left[x(t)+g\left(t, x_{t}\right)+B u(t)\right] d t+\int_{0}^{t} \gamma(t-s)\left[x(s)+g\left(s, x_{s}\right)\right] d s d t \\
& +f\left(t, x_{t}\right) d t+\sigma(t) d B^{H}(t), t \in[0, T], \\
\left.\Delta x\right|_{t-t_{k}}= & x\left(t_{k}^{+}\right)-x\left(t_{k}^{-}\right)=I_{k}\left(x\left(t_{k}^{-}\right)\right), \quad k=1, \ldots, m, \\
x(t)= & \varphi(t) \in \mathcal{L}_{2}^{0}\left(\Omega, \mathscr{B}_{h}\right), \text { for a.e. } t \in(-\infty, 0] .
\end{aligned}
$$

Here, $A$ is the infinitesimal generator of a strongly continuous semigroup of bounded linear operator $(T(t))_{t \geqslant 0}$ on a Hilbert space $X$ with domain $\mathscr{D}(A) ; \gamma(t)$ is a closed linear operator on $X$ with domain $\mathscr{D}(\gamma) \supset \mathscr{D}(\mathrm{A}) ; \mathrm{B}^{\mathrm{H}}$ is a fractional Brownian motion with Hurst parameter $\mathrm{H}>\frac{1}{2}$ on a real and separable Hilbert space $Y$; and the control function $u(\cdot)$ takes values in $\mathcal{L}^{2}([0, T], U)$, the Hilbert space of admissible control functions for a separable Hilbert space $U$; and B is a bounded linear operator from $U$ into $X$. The history $x_{t}:(-\infty, 0] \rightarrow X, x_{t}(\theta)=x(t+\theta)$, belongs to an abstract phase space $\mathscr{B}_{h}$ defined axiomatically, and $\mathrm{f}, \mathrm{g}:[0, \mathrm{~T}] \times \mathscr{B}_{\mathrm{h}} \rightarrow \mathrm{X}, \sigma:[0, \mathrm{~T}] \rightarrow \mathcal{L}_{2}^{0}(\mathrm{Y}, \mathrm{X})$ are appropriate functions, where $\mathcal{L}_{2}^{0}(\mathrm{Y}, \mathrm{X})$ denotes the space of all Q-Hilbert-Schmidt operators from $Y$ into $X$. Moreover, the fixed moments of time $t_{k}$ satisfy $0<t_{1}<t_{2}<\cdots<t_{m}<T, x\left(t_{k}^{-}\right)$and $x\left(t_{k}^{+}\right)$represent the left and right limits of $x(t)$ at time $t_{k}$, respectively. $\Delta x\left(t_{k}\right)$ denotes the jump in the state $x$ at time $t_{k}$ with I $: X \rightarrow X$ determining the size of the jump.

\section{Preliminaries}

In this section, we introduce the fractional Brownian motion as well as Wiener integral with respect to it. We also provide some important results which will bee needed throughout this paper. For more details on this section, we refer the reader to $[7,13,18]$.

Fix a time interval $[0, T]$ and let $(\Omega, \Im, P)$ be a complete probability space and $\left\{\beta^{\mathrm{H}}(t): t \in[0, T]\right\}$ be a one-dimensional fractional Brownian motion with Hurst parameter $H \in\left(\frac{1}{2}, 1\right)$. By definition, $\beta^{\mathrm{H}}$ is a centered Gaussian process with covariance function

$$
\mathrm{R}_{\mathrm{H}(\mathrm{t}, \mathrm{s})}=\mathbf{E}\left[\beta^{\mathrm{H}}(\mathrm{t}) \beta^{\mathrm{H}}(\mathrm{s})\right]=\frac{1}{2}\left(|\mathrm{t}|^{2 \mathrm{H}}+|\mathrm{s}|^{2 \mathrm{H}}-|\mathrm{t}-\mathrm{s}|^{2 \mathrm{H}}\right) .
$$

Moreover, $\beta^{\mathrm{H}}$ has the following Wiener integral representation

$$
\beta^{H}(t)=\int_{0}^{t} K_{H}(t, s) d \beta(s),
$$


where $\beta=\{\beta(t) ; t \in[0, T]\}$ is a Wiener process and kernel $K_{H}(t, s)$ is given by

$$
K_{H}(t, s)=c_{H} S^{\frac{1}{2}=H} \int_{0}^{t}(u-s)^{H-\frac{3}{2}} u^{H-\frac{1}{2}} d u,
$$

for $t>s$, where $c_{H}=\sqrt{\frac{H(2 H-1)}{g\left(2-2 \mathrm{H}, \mathrm{H}-\frac{1}{2}\right)}}$ and $g(\cdot, \cdot)$ denotes the Beta function. We take $K_{H}(t, s)=0$ if $t \leqslant s$. We will denote by $\mathscr{H}$ the reproducing kernel Hilbert space of the fractional Brownian motion. Precisely, $\mathscr{H}$ is the closure of set of indicator functions $\left\{1_{[0, t]}: t \in[0, T]\right\}$ with respect to the scalar product $\left\langle 1_{[0, t]}, 1_{[0, s]}\right\rangle_{\mathscr{H}}=\mathrm{R}_{\mathrm{H}}(\mathrm{t}, \mathrm{s})$.

The mapping $1_{[0, t]} \rightarrow \beta^{\mathrm{H}}(\mathrm{t})$ can be extended to an isometry between $\mathscr{H}$ and the first Wiener chaos and we will denote by $\beta^{\mathrm{H}}(\varphi)$ the image of $\varphi$ by the previous isometry.

Let $X$ and $Y$ be two real separable Hilbert spaces and let $\mathcal{L}(Y, X)$ be the space of bounded linear operator from $\mathrm{Y}$ to $\mathrm{X}$. Let $\mathrm{Q} \in \mathcal{L}(\mathrm{X}, \mathrm{Y})$ be an operator defined by $\mathrm{Q} e_{\mathrm{n}}=\lambda_{\mathrm{n}} e_{\mathrm{n}}$ with finite trace

$$
\operatorname{trQ}=\sum_{n=1}^{\infty} \lambda_{n}<\infty,
$$

where $\lambda_{n} \geqslant 0(n=1,2, \ldots)$ are non-negative real numbers and $\left\{e_{n}\right\}(n=1,2, \ldots)$ is a complete orthonormal basis in $\mathrm{Y}$. We define the infinte dimensional fractional Brownian motion on $\mathrm{Y}$ with covariance $\mathrm{Q}$ as

$$
\mathrm{B}^{\mathrm{H}}(\mathrm{t})=\mathrm{B}_{\mathrm{Q}}^{\mathrm{H}}(\mathrm{t})=\sum_{n=1}^{\infty} \sqrt{\lambda_{n}} e_{n} \beta_{n}^{\mathrm{H}}(\mathrm{t}) .
$$

where $\beta_{n}^{\mathrm{H}}$ are real, independent fractional Brownian motion's. This process is Gaussian, it starts from 0 , has zero mean and covariance

$$
E\left\langle B^{H}(t), x\right\rangle\left\langle B^{H}(s), y\right\rangle=R(s, t)\langle Q(x), y\rangle \text { for } x, y \in Y \text { and } t, s \in[0, T] .
$$

Now, define the Wiener integrals with respect to the Q-fractional Brownian motion, we introduce the space $\mathcal{L}_{2}^{0}=\mathcal{L}_{2}^{0}(\mathrm{Y}, \mathrm{X})$ of all Q-Hilbert-Schmidt operators $\zeta: \mathrm{Y} \rightarrow \mathrm{X}$. We recall that $\zeta \in \mathcal{L}(\mathrm{Y}, \mathrm{X})$ is called a Q-Hilbert-Schmidt operator, if

$$
\|\zeta\|_{\mathcal{L}_{2}^{0}}^{2}=\sum_{n=1}^{\infty}\left\|\sqrt{\lambda_{n}} \zeta e_{n}\right\|^{2}<\infty,
$$

and that the space $\mathcal{L}_{2}^{0}$ equipped with the inner product $\left\langle\varphi, \zeta>_{\mathcal{L}_{2}^{0}}=\sum_{n=1}^{\infty}<\varphi e_{n}, \zeta e_{n}>\right.$ is a separable Hilbert space. Let $\phi(s): s \in[0, T]$ be a function with values in $\mathcal{L}_{2}^{0}(Y, X)$ such that

$$
\sum_{n=1}^{\infty}\left\|K^{*} \phi Q^{1 / 2} e_{n}\right\|_{\mathcal{L}_{2}^{0}}^{2}<\infty .
$$

The Weiner integral of $\phi$ with respect to $\mathrm{B}^{\mathrm{H}}$ is defined by

$$
\int_{0}^{t} \phi(s) d B^{H}=\sum_{n=1}^{\infty} \int_{0}^{t} \sqrt{\lambda_{n}} \phi(s) e_{n} d \beta_{n}^{H}(s) .
$$

Lemma 2.1 ([13]). If $\zeta:[0, \mathrm{~T}] \rightarrow \mathcal{L}_{2}^{0}(\mathrm{Y}, \mathrm{X})$ satisfies $\int_{0}^{\mathrm{t}}\|\zeta(s)\|_{\mathcal{L}_{2}^{0}}^{2} \mathrm{~d} s<\infty$, then (2.1) is well defined as an X-valued random variable and

$$
\mathbf{E}\left\|\int_{0}^{\mathrm{t}} \zeta(\mathrm{s}) \mathrm{dB^{ \textrm {H } }}(\mathrm{s})\right\|^{2} \leqslant 2 \mathrm{H} \mathrm{t}^{2 \mathrm{H}-1} \int_{0}^{\mathrm{t}}\|\zeta\|_{\mathcal{L}_{2}^{0}}^{2} \mathrm{ds} .
$$


We assume that the phase space $\mathscr{B}_{\mathrm{h}}$ is a linear space of functions mapping $(-\infty, 0]$ into $\mathrm{X}$, endowed with a norm $\|\cdot\|_{\mathscr{B}_{h}}$. First, we present the abstract phase space $\mathscr{B}_{h}$. Assume that $h:(-\infty, 0] \rightarrow[0, \infty)$ is a continuous function with

$$
l=\int_{-\infty}^{0} h(s) d s<+\infty
$$

We define the abstract phase space $\mathscr{B}_{\mathrm{h}}$ by $\mathscr{B}_{\mathrm{h}}=\left\{\zeta:(-\infty, 0] \rightarrow \mathrm{X}\right.$ for any $\tau>0,\left(\mathbf{E}\|\zeta\|^{2}\right)^{1 / 2}$ is bounded and measurable function $[\tau, 0]$ and $\left.\int_{-\infty}^{0} h(t) \sup _{t \leqslant \tau \leqslant 0}\left(E\|\zeta(s)\|^{2}\right)^{1 / 2} d t<\infty\right\}$. If this space with the norm

$$
\|\zeta\|_{\mathscr{B}_{\mathrm{h}}}=\int_{-\infty}^{0} \mathrm{~h}(\mathrm{t}) \sup _{\mathrm{t} \leqslant s \leqslant 0}\left(\mathbf{E}\|\zeta\|^{2}\right)^{1 / 2} \mathrm{dt}
$$

then it is clear that $\left(\mathscr{B}_{\mathrm{h}},\|\cdot\|_{\mathscr{B}_{\mathrm{h}}}\right)$ is a Banach space.

We now consider the space $\mathscr{B}_{\mathscr{D} \mathrm{I}}\left(\mathscr{D}\right.$ and I stand for delay and impulse, respectively) given by $\mathscr{B}_{\mathscr{D} \mathrm{I}}=$ $\left\{x:(-\infty, \mathrm{T}] \rightarrow \mathrm{X}: x \mid \mathrm{I}_{\mathrm{k}} \in \mathscr{C}\left(\mathrm{I}_{\mathrm{k}}, \mathrm{X}\right)\right.$ and $x\left(\mathrm{t}_{\mathrm{k}}^{+}\right), x\left(\mathrm{t}_{\mathrm{k}}^{-}\right)$exist with $x\left(\mathrm{t}_{\mathrm{k}}^{+}\right)-x\left(\mathrm{t}_{\mathrm{k}}^{-}\right), \mathrm{k}=1,2, \ldots, \mathrm{m} \mathrm{x}_{0}-\varphi \in$ $\mathscr{B}_{h}$ and $\left.\sup _{0 \leqslant t \leqslant T} \mathbf{E}\left(\|x(t)\|^{2}\right)<\infty\right\}$, where $x \mid I_{k}$ is the restriction of $x$ to the interval $I_{k}=\left(t_{k}, t_{k+1}\right]$, $k=1,2, \ldots, m$. Then the function $\|\cdot\|_{\mathscr{B}_{h}}$ to be a semi-norm in $\mathscr{B}_{\mathscr{D}}$, it is defined by

$$
\|x\|_{\mathscr{B}_{\mathscr{D} \mathrm{I}}}=\left\|\mathrm{x}_{0}\right\|_{\mathscr{B}_{\mathrm{h}}}+\sup _{0<\mathrm{t}<\mathrm{T}}\left(\mathbf{E}\left(\|x(\mathrm{t})\|^{2}\right)\right)^{1 / 2} .
$$

The following lemma is a common property of phase spaces.

Lemma 2.2 ([13]). Suppose $\mathrm{x} \in \mathscr{B}_{\mathscr{D} \mathrm{I}}$, then for all $\mathrm{t} \in[0, \mathrm{~T}], \mathrm{x}_{\mathrm{t}} \in \mathscr{B}_{\mathrm{h}}$ and

$$
l\left(\mathbf{E}\left(\|x(t)\|^{2}\right)\right)^{\frac{1}{2}} \leqslant l \sup _{0 \leqslant s \leqslant t}\left(\mathbf{E}\|x(s)\|^{2}\right)^{\frac{1}{2}}+\left\|x_{0}\right\|_{\mathscr{B}_{h}}
$$

where $l=\int_{-\infty}^{0} h(s) d s<\infty$.

\subsection{Partial integrodifferential equations in Banach spaces}

Further, we recollect some basic results related to resolvent operators. Regarding the theory of resolvent operators, we refer the reader to [8]. Let $A$ and $\gamma(t)$ are closed linear operators on $X$ and $Y$ represents the Banach space $\mathscr{D}(A)$ equipped with the graph norm

$$
|y|_{Y}:=|A y|+|y| \text { for } y \in Y .
$$

The notation $\mathscr{C}([0, \infty) ; \mathrm{Y})$ stands for the space of all continuous functions from $[0, \infty)$ into $\mathrm{Y}$. We consider the following Cauchy problem

$$
\left\{\begin{array}{l}
v^{\prime}(t)=A v(t)+\int_{0}^{t} \gamma(t-s) v(s) d s \text { for } t \geqslant 0 \\
v(0)=v_{0} \in X
\end{array}\right.
$$

Definition 2.3 ([8]). A resolvent operator for equation (2.2) is a bounded linear operator valued function $\mathcal{R}(t) \in \mathcal{L}(X)$ for $t \geqslant 0$, satisfying the following properties:

(i) $\mathcal{R}(0)=I$ and $\|\mathcal{R}(t)\| \leqslant M e^{\lambda t}$ for some constants $M$ and $\beta$;

(ii) for each $x \in X, \mathcal{R}(t) x$ is strongly continuous for $t \geqslant 0$; 
(iii) for $x \in \mathrm{Y}, \mathcal{R}(\cdot) \mathrm{x} \in \mathscr{C}^{1}([0, \infty) ; \mathrm{X}) \bigcap \mathscr{C}([0, \infty) ; \mathrm{Y})$ and

$$
\mathcal{R}^{\prime}(\mathrm{t}) x=A \mathcal{R}(\mathrm{t}) x+\int_{0}^{t} \gamma(\mathrm{t}-\mathrm{s}) \mathcal{R}(s) x \mathrm{ds}=\mathcal{R}(\mathrm{t}) \mathrm{A} x+\int_{0}^{\mathrm{t}} \mathcal{R}(\mathrm{t}-\mathrm{s}) \mathrm{B}(\mathrm{s}) x \mathrm{ds} \text { for } \mathrm{t} \geqslant 0 .
$$

For additional details on resolvent operators, we refer the reader to [8]. In what follows we suppose the following assumptions.

(H1) The operator $A$ generator a $C_{0}$-semigroup $(T(t))_{t \geqslant 0}$ on $X$.

(H2) For all $t \geqslant 0, \gamma(t)$ is a continuous linear operator from $\left(Y,|\cdot|_{Y}\right)$ into $\left(X,|\cdot|_{X}\right)$. Moreover, there exists an integrable function $\mathscr{C}:[0, \infty) \rightarrow \mathbb{R}^{+}$such that for any $\mathrm{y} \in \mathrm{Y}, \mathrm{y} \rightarrow \gamma(\mathrm{t}) \mathrm{y}$ belongs to $\mathrm{W}^{1,1}([0, \infty) ; \mathrm{X})$ and

$$
\left|\frac{\mathrm{d}}{\mathrm{dt}} \gamma(\mathrm{t})(\mathrm{t}) \mathrm{y}\right|_{\mathrm{X}} \leqslant \mathscr{C}(\mathrm{t})|\mathrm{y}|_{\mathrm{Y}} \text { for } \mathrm{y} \in \mathrm{Y} \text { and } \mathrm{t} \geqslant 0
$$

Theorem 2.4 ([8]). Assume that hypotheses (H1) and (H2) hold. Then there exists a unique resolvent operator for the Cauchy problem (2.2).

Lemma 2.5 ([14]). For any $\mathrm{T}>0$ there exists a constant $\mathrm{L}=\mathrm{L}(\mathrm{T})$ such that

$$
\|\mathcal{R}(\mathrm{t}+\epsilon)-\mathcal{R}(\epsilon) \mathcal{R}(\mathrm{t})\|_{\mathrm{L}(\mathbb{X})} \leqslant \mathrm{L} \epsilon \text { for } 0 \leqslant \epsilon \leqslant \mathrm{t} \leqslant \mathrm{b} .
$$

In the sequel, we recall some results on existence of solutions for the following integrodifferential equation

$$
\left\{\begin{array}{l}
v^{\prime}(t)=A v(t)+\int_{0}^{t} \gamma(t-s) v(s) d s+q(t) \text { for } t \geqslant 0 \\
v(0)=v_{0} \in X
\end{array}\right.
$$

where $\mathrm{q}:[0, \infty) \rightarrow X$ is a continuous function.

Definition 2.6. A continuous function $v:[0, \infty) \rightarrow X$ is said to be a strict solution of equation (2.3) if

(i) $v \in \mathscr{C}^{1}([0, \infty) ; \mathrm{X}) \bigcap \mathscr{C}([0, \infty) ; \mathrm{Y})$;

(ii) $v$ satisfies equation (2.4) for $t \geqslant 0$.

Theorem 2.7 ([8]). Assume that (H1)-(H2) hold. If $v$ is a strict solution of Equation (2.3), then the following variation of constants formula holds

$$
v(t)=\mathcal{R}(t) v_{0}+\int_{0}^{t} \mathcal{R}(t-s) q(s) d s \text { for } t \geqslant 0
$$

Now, we have the following definition for mild solution of (2.3).

Definition 2.8. An $X$-valued process $\{x(t): t \in(-\infty, T]\}$ is a mild solution of (1.1) if

1. $x(t)$ is measurable for each $t>0, x(t)=\varphi(t)$ on $(\infty, 0]$,

$$
\left.\Delta x\right|_{t-t_{k}}=I_{k}\left(x\left(t_{k}^{-}\right)\right), k=1,2, \ldots, m,
$$

the restriction of $x(\cdot)$ to $[0, T]-\left\{t_{1}, t_{2}, \ldots, t_{m}\right\}$ is continuous;

2. for every $0 \leqslant s \leqslant t$, the process $x$ satisfies the following integral equation:

$$
\begin{aligned}
\chi(t)= & \mathcal{R}(t)[\varphi(0)-g(0, \varphi)]+g\left(t, x_{t}\right)+\int_{0}^{t} \mathcal{R}(t-s) f\left(s, x_{s}\right) d s+\int_{0}^{t} \mathcal{R}(t-s) B u(s) d s \\
& +\int_{0}^{t} \mathcal{R}(t-s) \sigma(s) d B^{H}(s)+\sum_{0<t_{k}<t} \mathcal{R}(t-s) I_{k}\left(x\left(t_{k}^{-}\right)\right), \quad P-\text { a.s.. }
\end{aligned}
$$


Definition 2.9. A function $v:[0, \infty) \rightarrow X$ is called a mild solution of (2.3) if $v$ satisfies the variation of constants formula (2.4) for $v_{0} \in \mathrm{X}$.

Definition 2.10. Let $V$ be a bounded closed and convex subset of a Banach space $X$ and let $\Pi_{1}, \Pi_{2}$ be two operators of $\mathrm{V}$ into $\mathrm{X}$ satisfying

(i) $\Pi_{1}(x)+\Pi_{2}(x) \in V$ whenever $x \in V$;

(ii) $\Pi_{1}$ is a contraction mapping; and

(iii) $\Pi_{2}$ is completely continuous.

Then, there exists $a z \in V$ such that $z=\Pi_{1}(z)+\Pi_{2}(z)$.

\section{Controllability result}

Definition 3.1. System (1.1) is said to be controllable on the interval $(-\infty, T]$ if for every initial stochastic process $\varphi$ defined on $(-\infty, T]$, there exists a stochastic control $u \in \mathcal{L}^{2}([0, T] ; U)$ such that the mild solution $x(\cdot)$ of $(1.1)$ satisfies $x(T)=x_{1}$.

In order to establish the controllability of (1.1), we impose the following hypotheses.

(H3) The resolvent operator $\mathcal{R}($.$) is compact and there exist constants M \geqslant 1$ such that $\|\mathcal{R}(t)\|^{2} \leqslant M$.

(H4) The mapping $\mathrm{g}:[0, \mathrm{~T}] \times \mathscr{B}_{\mathrm{h}} \rightarrow \mathrm{X}$ satisfies the following conditions and there exist constants $\mathrm{k}_{\mathrm{g}}>0$ such that

$$
\mathbf{E}\|g(t, x)-g(t, y)\|^{2} \leqslant k_{g}\|x-y\|_{\mathscr{B}_{h}}^{2}, t \in[0, T], x, y \in \mathscr{B}_{h}, \quad \overline{k_{g}}=\sup _{t \in[0, T]}\|g(t, 0)\|^{2} .
$$

(H5) The mapping $f:[0, T] \times \mathscr{B}_{h} \times X \rightarrow X$ satisfies the following conditions:

(i) the function $t \rightarrow f(t, x)$ is measurable for each $x \in \mathscr{B}_{h}$, the function $t \rightarrow f(t, x)$ is continuous for almost all $t \in[0, T]$;

(ii) for every positive integer $k$ there exists $p_{k} \in \mathcal{L}^{1}\left([0, T], \mathbb{R}^{+}\right)$, such that

$$
\|f(t, x)\|^{2} \leqslant p_{k}(t), \text { for all } t \in[0, T], \text { and } \lim _{k \rightarrow \infty} \frac{1}{k} \int_{0}^{T} p_{k}(\tau) d \tau=\gamma<\infty .
$$

(H6) The impulses functions $I_{k}$ for $k=1,2, \ldots, m$, satisfy the following condition. There exists positive constants $M_{k}, \widetilde{M_{k}}$ such that

$$
\left\|I_{k}(x)-I_{k}(y)\right\|^{2} \leqslant M_{k}\|x-y\|^{2} \text { and }\left\|I_{k}(x)\right\|^{2} \leqslant \widetilde{M_{k}} \text { for all } x, y \in \mathscr{B}_{h} .
$$

(H7) The function $\sigma:[0, \infty) \rightarrow \mathcal{L}_{2}^{0}(\mathrm{Y}, \mathrm{X})$ satisfies

$$
\int_{0}^{T}\|\sigma(s)\|_{\mathcal{L}_{2}^{0}}^{2} \mathrm{~d} s<\infty, \text { for } \mathrm{t}>0 .
$$

(H8) The linear operator $W$ from $U$ into $X$ defined by

$$
W u=\int_{0}^{T} \mathcal{R}(T-s) B u(s) d s
$$

has an inverse operator $W^{-1}$ that takes values in $\mathcal{L}^{2}([0, T], U) /$ ker $W$, where

$$
\operatorname{ker}(W)=\left\{x \in \mathcal{L}^{2}([0, T], U): W x=0\right\} .
$$

The main result of this paper is given in the next theorem. 
Theorem 3.2. Suppose that (H1)-(H8) hold. Then, the system (1.1) is controllable on $(-\infty, \mathrm{T}]$ provide that

$$
6 \mathrm{l}^{2}\left(1+7 \mathrm{MM}_{\mathrm{b}} \mathrm{M}_{\mathrm{W}} \mathrm{T}^{2}\right)\left[8\left[\mathrm{k}_{2}\left(1+2 \mathrm{k}_{1}\right)\right]+8 \mathrm{MT}^{2}\left[\mathrm{k}_{3}\left(1+2 \mathrm{k}_{4}\right)\right]\right]<1 .
$$

Proof. Using (H8) for an arbitrary function $x(\cdot)$, define the control

$$
\begin{aligned}
u_{x}(t)= & W^{-1}\left[x_{1}-\mathcal{R}(T)\left[\varphi(0)-g\left(0, x_{0}\right)\right]-g\left(T, x_{T}\right)-\int_{0}^{T} \mathcal{R}(T-s) f\left(s, x_{s}\right) d s\right. \\
& \left.-\int_{0}^{T} \mathcal{R}(T-s) \sigma(s) d B^{H}(s)-\sum_{0<t_{k}<t} \mathcal{R}\left(T-t_{k}\right) I_{k}\left(x\left(t_{k}^{-}\right)\right)\right](t) .
\end{aligned}
$$

Now, put the control $u(\cdot)$ into the stochastic control system (8) and obtain a nonlinear operator $\Gamma$ on $\mathscr{B}_{\mathscr{D} I}$ given by

$$
\Gamma(x)(t)=\left\{\begin{array}{l}
\varphi(t), \text { for } t \in(-\infty, 0], \\
\mathcal{R}(t)[\varphi(0)-g(0, \varphi, 0)]+g\left(t, x_{t}\right)+\int_{0}^{t} \mathcal{R}(t-s) B u_{x}(s) d s+\int_{0}^{t} \mathcal{R}(t-s) f\left(s, x_{s}\right) d s \\
+\int_{0}^{t} \mathcal{R}(t-s) \sigma(s) d B^{H}(s)+\sum_{0<t_{k}<t} \mathcal{R}\left(t-t_{k}\right) I_{k}\left(x\left(t_{k}^{-}\right)\right), \text {if } t \in[0, T] .
\end{array}\right.
$$

Then it is clear that to prove the existence of mild solutions to equations (1.1) is equivalent to find a fixed point for the operator. Clearly, $\Gamma \chi(T)=x_{1}$, which means that the control $u$ steers the system grom the initial state $\varphi$ to $x_{1}$ in time $T$, provided we can obtain a fixed point of the operator $\Gamma$ which implies that the system in controllable.

Let $y:(-\infty, T] \rightarrow X$ be the function defined by

$$
y(t)= \begin{cases}\varphi(t), & \text { if } t \in(-\infty, 0], \\ \mathcal{R}(t) \varphi(0), & \text { if } t \in[0, T],\end{cases}
$$

then, $y_{0}=\varphi$. For each function $z \in \mathscr{B}_{\mathscr{D}}$, set

$$
x(t)=z(t)+y(t) .
$$

It is obvious that $x$ satisfies the stochastic control system (2.5) if and only if $z$ satisfies $z_{0}=0$ and

$$
\begin{aligned}
z(t)= & g\left(t, z_{t}+y_{t}\right)-\mathcal{R}(t) g(0, \varphi)+\int_{0}^{t} \mathcal{R}(t-s) B_{z+y}(s) d s+\int_{0}^{t} R(t-s) f\left(s, z_{s}+y_{s}\right) d s \\
& +\int_{0}^{t} \mathcal{R}(t-s) \sigma(s) d B^{H}(s)+\sum_{0<t_{k}<t} R\left(t-t_{k}\right) I_{k}\left[z\left(t_{k}^{-}\right)-y\left(t_{k}^{-}\right)\right], \quad \text { if } t \in[0, T],
\end{aligned}
$$

where

$$
\begin{aligned}
u_{z+y}(t)= & W^{-1}\left[x_{1}-\mathcal{R}(T)\left[\varphi(0)-g\left(0, z_{0}+y_{0}\right)\right]-g\left(T, z_{T}+y_{T}\right)-\int_{0}^{T} \mathcal{R}(T-s) f\left(s, z_{s}+y_{s}\right) d s\right. \\
& \left.-\int_{0}^{T} \mathcal{R}(T-s) \sigma(s) d B^{H}(s)-\sum_{0<t_{k}<T} R\left(T-t_{k}\right) I_{k}\left[z\left(t_{k}^{-}\right)+y\left(t_{k}^{-}\right)\right]\right](t) .
\end{aligned}
$$

Set

$$
\mathscr{B}_{\mathscr{D} \mathrm{I}}^{0}=\left\{z \in \mathscr{B}_{\mathscr{D} \mathrm{I}}: z_{0}=0\right\},
$$


for any $z \in \mathscr{B}_{\mathscr{D} \mathrm{I}}^{0}$, we have

$$
\|z\|_{\mathscr{B}_{\mathscr{D}}^{0}}=\left\|z_{0}\right\|_{\mathscr{B}_{\mathrm{h}}}+\sup _{\mathrm{t} \in[0, \mathrm{~T}]}\left(\mathbf{E}\|z(\mathrm{t})\|^{2}\right)^{\frac{1}{2}}=\sup _{\mathrm{t} \in[0, \mathrm{~T}]}\left(\mathbf{E}\|z(\mathrm{t})\|^{2}\right)^{\frac{1}{2}}
$$

Then, $\left(\mathscr{B}_{\mathscr{D} I}^{0},\|\cdot\|_{\mathscr{B}_{\mathscr{D}}}^{0}\right)$ is a Banach space. Define the operator $\Theta: \mathscr{B}_{\mathscr{D} \mathrm{I}}^{0} \rightarrow \mathscr{B}_{\mathscr{D} \mathrm{I}}^{0}$ by

$$
(\Theta z)(t)=\left\{\begin{array}{l}
0, \quad \text { if } t \in(-\infty, 0], \\
g\left(t, z_{t}+y_{t}\right)-\mathcal{R}(t) g(0, \varphi)+\int_{0}^{t} \mathcal{R}(t-s) B_{z+y}(s) d s+\int_{0}^{t} \mathcal{R}(t-s) f\left(s, z_{s}+y_{s}\right) d s \\
\quad+\int_{0}^{t} \mathcal{R}(t-s) \sigma(s) d B^{H}(s)+\sum_{0<t_{k}<t} \mathcal{R}\left(t-t_{k}\right) I_{k}\left[z\left(t_{k}^{-}\right)-y\left(t_{k}^{-}\right)\right], \quad \text { if } t \in[0, T],
\end{array}\right.
$$

Set

$$
\mathscr{B}_{k}=\left\{z \in \mathscr{B}_{\mathscr{D} \mathrm{I}}^{0}:\|z\|_{\mathscr{B}_{\mathscr{D}}^{0}}^{2} \leqslant k\right\} \text {, for some } k \geqslant 0 \text {, }
$$

then $\mathscr{B}_{\mathrm{k}} \subseteq \mathscr{B}_{\mathscr{D} \mathrm{I}}^{0}$ is a bounded closed convex set, and for $z \in \mathscr{B}_{\mathrm{k}}$, we have

$$
\begin{aligned}
\left\|z_{\mathrm{t}}+y_{\mathrm{t}}\right\|_{\mathscr{B}_{\mathscr{D} \mathrm{I}}} & \leqslant 2\left(\left\|z_{\mathrm{t}}\right\|_{\mathscr{B}_{\mathscr{D} \mathscr{I}}}^{2}+\left\|y_{\mathrm{t}}\right\|_{\mathscr{B}_{\mathscr{D} \mathscr{I}}}^{2}\right) \\
& \leqslant 4\left(\mathrm{l}^{2} \sup _{0 \leqslant s \leqslant \mathrm{t}} \mathrm{E}\|z(s)\|^{2}+\left\|z_{0}\right\|_{\mathscr{B}_{\mathrm{h}}}^{2}+\mathrm{l}^{2} \sup _{0 \leqslant s \leqslant \mathrm{t}} \mathrm{E}\|\mathrm{y}(\mathrm{s})\|^{2}+\left\|\mathrm{y}_{0}\right\|_{\mathscr{B}_{\mathrm{h}}}^{2}\right) \\
& \leqslant 4 l^{2}\left(\mathrm{k}+\mathrm{ME}\|\varphi(0)\|^{2}\right)+4\|y\|_{\mathscr{B}_{\mathrm{h}}}^{2}:=\mathrm{r}^{*} .
\end{aligned}
$$

Next,

$$
\begin{aligned}
\mathbf{E}\left\|\mathrm{u}_{z+y}\right\|^{2} \leqslant & 7 \mathrm{M}_{W}\left[\left\|\mathrm{x}_{1}\right\|^{2}+\mathrm{ME}\|\varphi(0)\|^{2}+2 \mathrm{M}\left[\mathrm{k}_{\mathrm{g}}\|y\|_{\mathscr{B}_{\mathrm{h}}}^{2}+\overline{\mathrm{k}_{\mathrm{g}}}\right]+\mathrm{MT} \int_{0}^{\mathrm{t}} \mathrm{pr}^{*}(\mathrm{~s}) \mathrm{ds}\right. \\
& \left.+2 \mathrm{MT}^{2 \mathrm{H}-1} \int_{0}^{\mathrm{T}}\|\sigma(\mathrm{s})\|_{\mathcal{L}_{2}^{0}}^{2} \mathrm{~d} \mathrm{~s}+\mathrm{mM} \sum_{\mathrm{k}=1}^{\mathrm{m}} \widetilde{M}_{\mathrm{k}}\right]:=\mathcal{G} .
\end{aligned}
$$

It is clear that the operator $\Theta$ has a fixed point if and only if $\hat{\Theta}$ has one, so it turns to prove that $\hat{\Theta}$ has a fixed point. To this end, we decompose $\hat{\Theta}$ as $\hat{\Theta}=\Theta_{1}+\Theta_{2}$, where $\Theta_{1}$ and $\Theta_{2}$ are defined on $\mathscr{B}_{\mathscr{D}}^{0}$, respectively by

$$
\begin{aligned}
& \left(\Theta_{1} z\right)(t)= \begin{cases}0, & \text { if } t \in(-\infty, 0], \\
g\left(t, z_{t}+y_{t}\right)-\mathcal{R}(t) g(0, \varphi)+\int_{0}^{t} \mathcal{R}(t-s) \sigma(s) d B^{H}(s), & \text { if } t \in[0, T],\end{cases} \\
& \left(\Theta_{2} z\right)(t)= \begin{cases}0, & \text { if } t \in(-\infty, 0], \\
\int_{0}^{t} \mathcal{R}(t-s) f\left(s, z_{s}+y_{s}\right) d s+\int_{0}^{t} \mathcal{R}(t-s) B u_{z+y}(s) d s & \text { if } t \in[0, T],\end{cases}
\end{aligned}
$$

In order to apply the Krasnoselskii fixed point theorem for the operator $\hat{\Theta}$, we prove the following assertions:

(1) $\Theta_{1}(x)+\Theta_{2}(x) \in \mathscr{B}_{k}$ whenever $x \in \mathscr{B}_{k}$;

(2) $\Theta_{1}$ is a contraction; 
(3) $\Theta_{2}$ is continuous and compact map.

For the sake of convenience, the proof will be given in several steps.

Step 1: We claim that there exists a positive number k, such that $\Theta_{1}(x)+\Theta_{2}(x) \in \mathscr{B}_{k}$ whenever $x \in \mathscr{B}_{k}$. If it is not true, then for each positive number $k$, there is a function $z^{k}(\cdot) \in \mathscr{B}_{k}$, but $\Theta_{1}\left(z^{k}\right)+\Theta_{2}\left(z^{k}\right) \notin \mathscr{B}_{k}$, that is

$$
\mathbf{E}\left\|\Theta_{1}\left(z^{k}\right)(t)+\Theta_{2}\left(z^{k}\right)(t)\right\|^{2}>k \text { for } t \in[0, T] .
$$

However, on the other hand, we have

$$
\begin{aligned}
& k<\mathbf{E}\left\|\Theta_{1}\left(z^{k}\right)(t)+\Theta_{2}\left(z^{k}\right)(t)\right\|^{2} \leqslant 6\left[2 M\left[k_{g}\|y\|_{\mathscr{B}_{h}}^{2}+\overline{k_{g}}\right]+2\left[r^{*}+\overline{k_{g}}\right] M M_{b} T^{2} \mathcal{G}\right. \\
& \left.+M T \int_{0}^{T} \operatorname{pr}^{*}(s) d s+2 M T^{2 H-1} \int_{0}^{T}\|\sigma(s)\|_{\mathcal{L}_{2}^{0}}^{2} d s+M \sum_{k=1}^{m} \tilde{M}_{k}\right] \\
& \leqslant 6\left(1+6 M^{b} M_{w} T^{2}\right)\left[2 M\left[k_{g}\|y\|_{\mathscr{B}_{h}}^{2}+\overline{k_{g}}\right]+2\left[r^{*}+\bar{k}_{g}\right] M M_{b} T^{2} \mathcal{G}\right. \\
& \left.+M T \int_{0}^{T} \operatorname{pr}^{*}(s) d s+2 M T^{2 H-1} \int_{0}^{T}\|\sigma(s)\|_{\mathcal{L}_{2}^{0}}^{2} d s+M \sum_{k=1}^{m} \tilde{M}_{k}\right] \\
& +6 M M_{b} M_{w} T^{2}\left[\left\|x_{1}\right\|^{2}+M E\|\varphi(0)\|^{2}\right] \\
& \leqslant \overline{\mathrm{K}}+6\left(1+6 \mathrm{MM}_{\mathrm{b}} \mathrm{M}_{w} \mathrm{~T}^{2}\right)\left[2 \mathrm{MT} \int_{0}^{\mathrm{T}} \mathrm{pr}^{*}(\mathrm{~s}) \mathrm{d} s\right] \text {, }
\end{aligned}
$$

where

$$
\begin{aligned}
\overline{\mathrm{K}}= & 6\left(1+6 \mathrm{MM}_{\mathrm{b}} \mathrm{M}_{w} \mathrm{~T}^{2}\right)\left[2 \mathrm{M}\left[\mathrm{k}_{\mathrm{g}}\|y\|_{\mathscr{B}_{\mathrm{h}}}^{2}+\overline{\mathrm{k}_{\mathrm{g}}}\right]+2 \overline{\mathrm{k}_{\mathrm{g}}}+2 M \mathrm{~T}^{2 \mathrm{H}-1} \int_{0}^{\mathrm{T}}\|\sigma(\mathrm{s})\|_{\mathcal{L}_{2}^{0}}^{2} \mathrm{ds}\right. \\
& \left.+\mathrm{M} \sum_{\mathrm{k}=1}^{\mathrm{m}} \tilde{\mathrm{M}}_{\mathrm{k}}+6 \mathrm{MM} \mathrm{M}_{\mathrm{b}} \mathrm{M}_{w} \mathrm{~T}^{2}\left[\left\|\mathrm{x}_{1}\right\|^{2}+\mathrm{ME}\|\varphi(0)\|^{2}\right]\right]
\end{aligned}
$$

is independent of $k$. Dividing both sides by $k$ and taking the lower limit as $k \rightarrow \infty$, we obtain

$$
\begin{aligned}
r^{*} & =4 l^{2}\left[k+M E\|\varphi(0)\|^{2}\right]+4\|y\|_{\mathscr{B}_{h}} \rightarrow \infty \text { as } k \rightarrow \infty . \\
\lim _{k \rightarrow \infty} \inf \frac{\int_{0}^{t} p r^{*}(s) d s}{k} & =\lim _{k \rightarrow \infty} \inf \frac{\int_{0}^{t} \operatorname{pr}^{*}(s) d s}{r^{*}} \cdot \frac{r^{*}}{k}=4 l^{2} \gamma .
\end{aligned}
$$

Thus, we have

$$
6 l^{2}\left(1+6 M M_{b} M_{w} T^{2}\right)[8 M T \gamma] \geqslant 1 .
$$

This contradicts (3.1). Hence $\left(\Theta_{1}+\Theta_{2}\right)\left(\mathscr{B}_{\mathrm{k}}\right) \subseteq \mathscr{B}_{\mathrm{k}}$.

Step 2: $\Theta_{1}$ is a contraction. Let $\mathrm{t} \in[0, \mathrm{~T}]$ and $z^{1}, z^{2} \in \mathscr{B}_{\mathscr{D} \mathrm{I}}^{0}$

$$
\begin{aligned}
\mathbf{E}\left\|\left(\Theta_{1} z^{1}\right)(\mathrm{t})-\left(\Theta_{1} z^{2}\right)(\mathrm{t})\right\|^{2} & \leqslant 2 \mathbf{E}\left\|g\left(t, z_{\mathrm{t}}^{1}+y_{\mathrm{t}}\right)-\mathrm{g}\left(\mathrm{t}, z_{\mathrm{t}}^{2}+\mathrm{y}_{\mathrm{t}}\right)\right\|^{2} \\
& \leqslant k_{\mathrm{g}}\left\|z_{\mathrm{t}}^{1}+y_{\mathrm{t}}-z_{\mathrm{t}}^{2}+\mathrm{y}_{\mathrm{t}}\right\|^{2} \\
& \leqslant k_{\mathrm{g}}\left\|z_{\mathrm{t}}^{1}-z_{\mathrm{t}}^{2}\right\|^{2}
\end{aligned}
$$




$$
\begin{aligned}
& \leqslant k_{\mathrm{g}} \times\left[2 \mathrm{l}^{2} \sup _{0 \leqslant s \leqslant \mathrm{~T}} \mathrm{E}\left\|z^{1}(\mathrm{~s})-z^{2}(\mathrm{~s})\right\|^{2}+2\left(\left\|z_{0}^{1}\right\|_{\mathscr{B}_{\mathrm{h}}}^{2}+\left\|z_{0}^{2}\right\|_{\mathscr{B}_{\mathrm{h}}}^{2}\right)\right] \\
& \leqslant \Gamma \times \sup _{0 \leqslant s \leqslant \mathrm{~T}} \mathbf{E}\left\|z_{\mathrm{s}}^{1}-z_{\mathrm{s}}^{2}\right\|^{2},
\end{aligned}
$$

where $\Gamma=2 k_{\mathrm{g}} \mathrm{l}^{2}<1$. Thus $\Theta_{1}$ is a contraction on $\mathscr{B}_{\mathscr{D} \mathrm{I}}^{0}$.

Step 3: $\Theta_{2}$ is completely continuous on $\mathscr{B}_{\mathscr{D} \mathrm{I}}^{0}$.

Claim 1. $\Theta_{2}$ is continuous on $\mathscr{B}_{\mathscr{D}}^{0}$. Let $z^{n}$ be a sequence such that $z^{n} \rightarrow z$ in $\mathscr{B}_{\mathscr{D} \mathrm{I}}^{0}$. Then there existes a number $\mathrm{k}>0$ such that $\left\|z^{\mathfrak{n}}(\mathrm{t})\right\| \leqslant \mathrm{k}$, for all $\mathrm{n}$ and a.c. $\mathrm{t} \in[0, \mathrm{~T}]$, so $z^{\mathfrak{n}} \in \mathscr{B}$ and $z \in \mathscr{B}$. By hypothesis (H5)-(H6), we have

(1) $I_{k}, k=1,2 \ldots, m$ is continuous;

(2) $f\left(t, z_{t}^{n}+y_{t}\right) \rightarrow f\left(t, z_{t}+y_{t}\right)$ for each $t \in[0, T]$, since

$$
\left\|f\left(t, z_{t}^{n}+y_{t}\right)-f\left(t, z_{t}+y_{t}\right)\right\|^{2} \leqslant 2 p r^{*}(t) .
$$

From (H4), Holder's inequality and the dominated convergence theorem, we have

$$
\begin{aligned}
E\left\|\Theta_{2} z^{n}(t)-\left(\Theta_{2} z\right)(t)\right\|^{2} \leqslant & 3 E\left\|\int_{0}^{t} \mathcal{R}(t-s) B\left[u_{z^{n}+y}-u_{z+y}\right] d s\right\|^{2} \\
& +3 E\left\|\int_{0}^{t} \mathcal{R}(t-s)\left[f\left(s, z_{s}^{n}+y_{s}\right)-f\left(s, z_{s}+y_{s}\right)\right] d s\right\|^{2} \\
& +3 E\left\|\sum_{0 \leqslant t_{k} \leqslant t} \mathcal{R}\left(t-t_{k}\right)\left[I_{k}\left(z^{n}\left(t_{k}^{-}\right)+y\left(t_{k}^{-}\right)\right)-I_{k}\left(z\left(t_{k}^{-}\right)+y\left(t_{k}^{-}\right)\right)\right]\right\|^{2} \|^{T} \\
\leqslant & 9 M_{w} M_{b} M T \int_{0}^{T}\left[E\left\|g\left(T, z_{T}^{n}+y_{T}\right)-g\left(T, z_{T}+y_{T}\right)\right\|^{2}\right. \\
& +M T \int_{0}^{T} E\left\|f\left(s, z_{s}^{n}+y_{s}\right)-f\left(s, z_{s}+y_{s}\right)\right\|^{2} d s \\
& \left.+M m \sum_{k=1}^{m} E\left\|I_{k}\left(z^{n}\left(t_{k}^{-}\right)+y\left(t_{k}^{-}\right)\right)-I_{k}\left(z\left(t_{k}^{-}\right)+y\left(t_{k}^{-}\right)\right)\right\|^{2}\right](\lambda) d \lambda \\
& +3 M T \int_{0}^{T} E\left\|f\left(s, z_{s}^{n}+y_{s}\right)-f\left(s, z_{s}+y_{s}\right)\right\|^{2} d s \\
& +3 m M \sum_{k=1}^{m} E\left\|I_{k}\left(z^{n}\left(t_{k}^{-}\right)+y\left(t_{k}^{-}\right)\right)-I_{k}\left(z\left(t_{k}^{-}\right)+y\left(t_{k}^{-}\right)\right)\right\|^{2} \\
\rightarrow & 0 \text { as } n \rightarrow \infty .
\end{aligned}
$$

Thus, $\Theta_{2}$ is continuous.

Claim 2. $\Theta_{2}$ maps $\mathscr{B}_{k}$ into equicontinuous family. Let $z \in \mathscr{B}_{k}$ and $\tau_{1}, \tau_{2} \in[0, T], \tau_{1}, \tau_{2} \in t_{k}, k=1, \ldots, m$, we have

$$
\begin{aligned}
\mathrm{E}\left\|\left(\Theta_{2} z\right)\left(\tau_{2}\right)-\left(\Theta_{2} z\right)\left(\tau_{1}\right)\right\|^{2} \leqslant & 6 \mathrm{E}\left\|\int_{0}^{\tau_{1}}\left[\mathcal{R}\left(\tau_{2}-s\right)-\mathcal{R}\left(\tau_{1}-s\right)\right] \mathrm{f}\left(\mathrm{s}, z_{\mathrm{s}}+\mathrm{y}_{\mathrm{s}}\right) \mathrm{ds}\right\|^{2} \\
& +6 \mathrm{E}\left\|\int_{0}^{\tau_{1}}\left[\mathcal{R}\left(\tau_{2}-s\right)-\mathcal{R}\left(\tau_{1}-s\right)\right] \mathrm{Bu}(\mathrm{s}) \mathrm{ds}\right\|^{2} \\
& +6 \mathrm{E}\left\|\sum_{0<\mathrm{t}_{\mathrm{k}}<\mathrm{t}}\left[\mathcal{R}\left(\tau_{2}-\mathrm{t}_{\mathrm{k}}\right)-\mathcal{R}\left(\tau_{1}-\mathrm{t}_{\mathrm{k}}\right)\right] \mathrm{I}_{\mathrm{k}}\left(z\left(\mathrm{t}_{\mathrm{k}}^{-}\right)+\mathrm{y}\left(\mathrm{t}_{\mathrm{k}}^{-}\right)\right)\right\|^{2}
\end{aligned}
$$




$$
\begin{aligned}
& +6 \mathbf{E}\left\|\int_{\tau_{1}}^{\tau_{2}} \mathcal{R}\left(\tau_{2}-s\right) f\left(s, z_{s}+y_{s}\right) d s\right\|^{2}+6 E\left\|\int_{\tau_{1}}^{\tau_{2}} \mathcal{R}\left(\tau_{2}-s\right) B u(s) d s\right\|^{2} \\
& +6 E\left\|\sum_{\tau_{1}<t_{k}<\tau_{2}}\left[\mathcal{R}\left(\tau_{2}-t_{k}\right)\right] I_{k}\left(z\left(t_{k}^{-}\right)+y\left(t_{k}^{-}\right)\right)\right\|^{2} .
\end{aligned}
$$

From (3.2) and Holder's inequality, it follows that

$$
\begin{aligned}
\mathrm{E}\left\|\left(\Theta_{2} z\right)\left(\tau_{2}\right)-\left(\Theta_{2} z\right)\left(\tau_{1}\right)\right\|^{2} \leqslant & 6 \mathrm{~T}\left\|\int_{0}^{\tau_{1}}\right\| \mathcal{R}\left(\tau_{2}-s\right)-\mathcal{R}\left(\tau_{1}-s\right)\|\|^{2} \operatorname{pr}^{*}(\mathrm{~s}) \mathrm{ds} \\
& +6 \mathrm{TM} \mathrm{b} \mathcal{G} \int_{0}^{\tau_{1}}\left\|\mathcal{R}\left(\tau_{2}-s\right)-\mathcal{R}\left(\tau_{1}-s\right)\right\|^{2} \mathrm{ds} \\
& +6 \mathrm{~m} \sum_{0<\mathrm{t}_{\mathrm{k}}<\tau_{1}}^{\tau_{1}}\left\|\mathcal{R}\left(\tau_{2}-\mathrm{t}_{\mathrm{k}}\right)-\mathcal{R}\left(\tau_{1}-\mathrm{t}_{\mathrm{k}}\right)\right\|^{2} \tilde{M}_{\mathrm{k}} \\
& +6 \mathrm{~T} \int_{\tau_{1}}^{\tau_{2}}\left\|\mathcal{R}\left(\tau_{2}-\mathrm{s}\right)\right\|^{2} \operatorname{pr}^{*}(\mathrm{~s}) \mathrm{ds}+6 \mathrm{TM}_{\mathrm{b}} \mathcal{G} \int_{\tau_{1}}^{\tau_{2}}\left\|\mathcal{R}\left(\tau_{2}-s\right)\right\|^{2}+6 \mathrm{mM} \sum_{\tau_{1}<\mathrm{t}_{\mathrm{k}}<\tau_{2}} \tilde{M}_{\mathrm{k}} .
\end{aligned}
$$

The right-hand side is independent of $z \in \mathscr{B}_{k}$ and tends to zero as $\tau_{2}-\tau_{1} \rightarrow 0$, since the compactness of $\mathcal{R}(t)_{t>0}$ implies the continuity in the uniform operator topology. Thus, $\Theta_{2}$ maps $\mathscr{B}_{k}$ into an equicontinuous family of functions. The equicontinuous for the cases $\tau_{1}<\tau_{2} \leqslant 0$ and $\tau_{1}<0<\tau_{2}$ are obvious.

Claim 3. $\left(\Theta_{2} \mathscr{B}_{k}\right)(t)$ is precompact set in $\mathrm{X}$. Let $0<\mathrm{t} \leqslant \mathrm{T}$ be fixed, $0<\epsilon<\mathrm{t}$, for $z \in \mathscr{B}_{\mathrm{k}}$, we define

$$
\begin{aligned}
\left(\Theta_{2, \epsilon} z\right)(t)= & \mathcal{R}(\epsilon) \int_{0}^{t-\epsilon} \mathcal{R}(\mathrm{t}-\mathrm{s}-\epsilon) \mathrm{f}\left(\mathrm{s}, z_{\mathrm{s}}+\mathrm{y}_{\mathrm{s}}\right) \mathrm{d} \mathrm{s}+\mathcal{R}(\epsilon) \int_{0}^{\mathrm{t}-\epsilon} \mathcal{R}(\mathrm{t}-\mathrm{s}-\epsilon) \mathrm{Bu} u_{z+y}(\mathrm{~s}) \mathrm{d} s \\
& +\mathcal{R}(\epsilon) \sum_{0<\mathrm{t}_{\mathrm{k}}<\mathrm{t}-\epsilon} \mathcal{R}\left(\mathrm{t}-\mathrm{t}_{\mathrm{k}}-\epsilon\right) \mathrm{I}_{\mathrm{k}}\left(z\left(\mathrm{t}_{\mathrm{k}}^{-}\right)+\mathrm{y}\left(\mathrm{t}_{\mathrm{k}}^{-}\right)\right) .
\end{aligned}
$$

and

$$
\begin{aligned}
\left(\tilde{\Theta}_{2, \epsilon} z\right)(t)= & \int_{0}^{t-\epsilon} \mathcal{R}(t-s) f\left(s, z_{s}+y_{s}\right) d s+\int_{0}^{t-\epsilon} \mathcal{R}(t-s) B u_{z+y}(s) d s \\
& +\sum_{0<t_{k}<t} \mathcal{R}\left(t-t_{k}\right) I_{k}\left(z\left(t_{k}^{-}\right)+y\left(t_{k}^{-}\right)\right) .
\end{aligned}
$$

Using the estimation (3.2) as above and by the compactness of $\mathcal{R}(t)_{t>0}$, we obtain $V_{\epsilon}(t)=\left\{\left(\Theta_{2, \epsilon} z\right)(t): z \in\right.$ $\left.\mathscr{B}_{\mathrm{k}}\right\}$ is relative compact in $\mathrm{X}$ for every $\epsilon, 0<\epsilon<\mathrm{t}$. Moreover, also by Lemma 2.5 and Holder inequality, for each $z \in \mathscr{B}_{k}$, we obtain

$$
\begin{aligned}
\mathbf{E}\left\|\left(\Theta_{2, \epsilon} z\right)(\mathrm{t})-\left(\tilde{\Theta}_{2, \epsilon} z\right)(\mathrm{t})\right\|^{2} \leqslant & 3 \mathrm{~T} \int_{0}^{\mathrm{t}-\epsilon}\|\mathcal{R}(\epsilon) \mathcal{R}(\mathrm{t}-\mathrm{s}-\epsilon)-\mathcal{R}(\mathrm{t}-\mathrm{s})\|_{\mathcal{L}(\mathrm{X})}^{2} \mathbf{E}\left\|\mathrm{f}\left(\mathrm{s}, z_{\mathrm{s}}+\mathrm{y}_{\mathrm{s}}\right)\right\|^{2} \mathrm{ds} \\
& +3 \mathrm{TM}_{\mathrm{b}} \mathcal{G} \int_{0}^{\mathrm{t}-\epsilon}\|\mathcal{R}(\epsilon) \mathcal{R}(\mathrm{t}-\mathrm{s}-\epsilon)-\mathcal{R}(\mathrm{t}-\mathrm{s})\|_{\mathcal{L}(\mathrm{X})}^{2} \mathrm{ds} \\
& +3 \mathrm{~m} \sum_{\mathrm{t}-\epsilon<\mathrm{t}_{\mathrm{k}}<\mathrm{t}}\left\|\mathcal{R}(\epsilon) \mathcal{R}\left(\mathrm{t}-\mathrm{t}_{\mathrm{k}}-\epsilon\right)-\mathcal{R}\left(\mathrm{t}-\mathrm{t}_{\mathrm{k}}\right)\right\|_{\mathcal{L}(\mathrm{X})}^{2} \mathbf{E}\left\|\mathrm{I}_{\mathrm{k}}\left(z\left(\mathrm{t}_{\mathrm{k}}^{-}\right)+\mathrm{y}\left(\mathrm{t}_{\mathrm{k}}^{-}\right)\right)\right\|^{2} .
\end{aligned}
$$

So the set $\tilde{V}_{\epsilon}(t)=\left\{\left(\tilde{\Theta}_{2, \epsilon} z\right)(t): z \in \mathscr{B}_{k}\right\}$ is precompact in $\mathrm{X}$ by using the total boundedness. Then for $z \in \mathscr{B}_{k}$, we have

$$
\mathbf{E}\left\|\left(\Theta_{2} z\right)(t)-\left(\tilde{\Theta}_{2, \epsilon} z\right)(t)\right\|^{2} \leqslant 3 T \int_{t-\epsilon}^{t}\|\mathcal{R}(t-s)\|^{2} E\left\|f\left(s, z_{s}+y_{s}\right)\right\|^{2} d s+3 T M_{b} \mathcal{G} \int_{t-\epsilon}^{t}\|\mathcal{R}(t-s)\|^{2} d s
$$




$$
\begin{aligned}
& +3 m \sum_{t-\epsilon<t_{k}<t}\left\|\mathcal{R}\left(t-t_{k}\right)\right\|^{2} E\left\|I_{k}\left(z\left(t_{k}^{-}\right)+y\left(t_{k}^{-}\right)\right)\right\|^{2} \\
\leqslant & 3 T M \int_{t-\epsilon}^{t} \operatorname{pr}^{*}(s) d s+3 T M_{b} \mathcal{G M} \epsilon+3 m M \sum_{t-\epsilon<t_{k}<t} \tilde{M}_{k} .
\end{aligned}
$$

Therefore,

$$
\mathbf{E}\left\|\left(\Theta_{2} z\right)(\mathbf{t})-\left(\tilde{\Theta}_{2, \epsilon} z\right)(t)\right\|^{2} \rightarrow 0, \quad \text { as } \epsilon \rightarrow 0^{+},
$$

and there are precompact sets arbitrarily close to the set $\mathrm{V}(\mathrm{t})=\left\{\left(\Theta_{2, \epsilon} z\right)(\mathrm{t}): z \in \mathscr{B}_{\mathrm{k}}\right\}$, hence the set $\mathrm{V}(\mathrm{t})$ is also precompact in $X$. Thus, by Arzela-Ascoli theorem $\Theta_{2}$ is compact. Thus, by Krasnoselskii fixed point theorem there exists a fixed point $z\left(\right.$.) for $\hat{\Theta}$ on $\mathscr{B}_{k}$. If we define $x(t)=z(t)+y(t),-\infty<t \leqslant T$, it is easy to say that $x($.$) is a mild solution of (1.1) satisfying x_{0}=\varphi, x(T)=x_{1}$. Hence the proof is completed.

\section{Application}

We consider the neutral impulsive stochastic functional integrodifferential equations with infinite delays, driven by a fractional Brownian motion of the form:

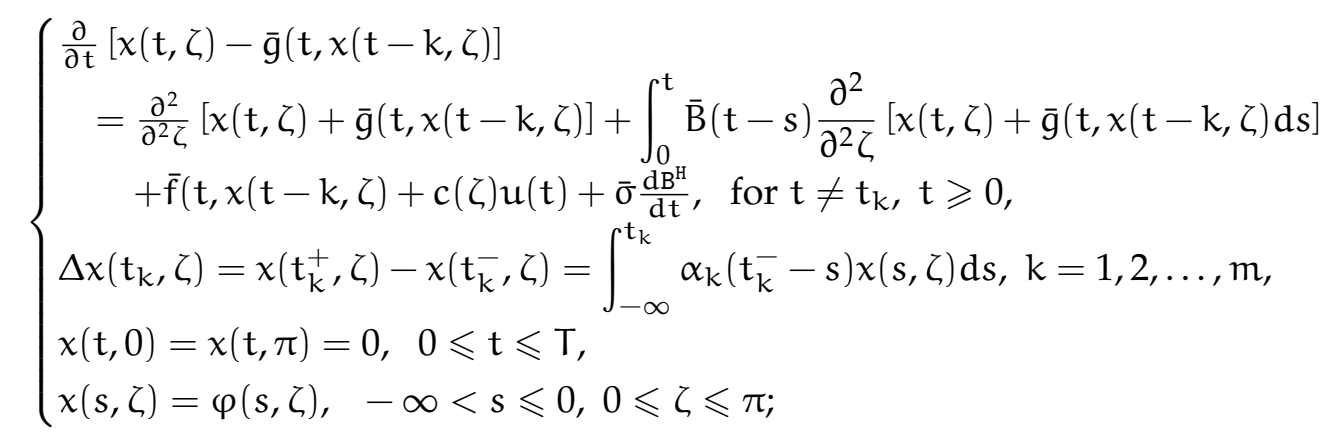

where $\mathrm{B}^{\mathrm{H}}(\mathrm{t})$ is cylindrical fractional Brownian motion, and $\varphi:(-\infty, 0] \times[0, \pi] \rightarrow \mathbb{R}$ is a given continuous stochastic process such that $\|\varphi\|_{\mathscr{B}_{h}}^{2}<\infty$. We take $\mathrm{X}=\mathrm{Y}=\mathrm{U}=\mathcal{L}^{2}([0, \pi])$ with norm $\|\cdot\|$. Define the operator $A: D(A) \subset X \rightarrow X$ given by $A=\frac{\partial^{2}}{\partial^{2} \zeta}$ with $D(A)=\left\{y \in X: y^{\prime}\right.$ is absolutely continuous $y^{\prime \prime} \in$ $X, y(0)=y(\pi)=0\}$, then we get

$$
A x=\sum_{n=1}^{\infty} n^{2}<x, e_{n}>e_{n}, \quad x \in D(A),
$$

where $e_{n}=\sqrt{\frac{2}{\pi}} \sin n x, n=1,2, \ldots$ is an orthogonal set of eigenfunctions of $A$. We choose the phase function $h(s)=e^{4 s}, s<0$, then $l=\int_{-\infty}^{0} h(s) d s=1 / 4<\infty$ and the abstract phase space $\mathscr{B}_{h}$ is Banach space with the norm

$$
\|\varphi\|_{\mathscr{B}_{\mathrm{h}}}=\int_{-\infty}^{0} h(s) \sup _{\theta \in[s, 0]} \mathbf{E}\left(\|\varphi(\theta)\|^{2}\right)^{1 / 2} \mathrm{ds}
$$

To rewrite the initial-boundary value problem (4.1) in the abstract from we assume the following.

For $(t, \varphi) \in[0, T] \times \mathscr{B}_{h}$, where $\varphi(\theta)(\zeta)=\varphi(\theta, \zeta),(\theta, \zeta) \in(-\infty, 0] \times[0, \pi]$, we put $\chi(t)(\zeta)=\chi(t, \zeta)$. The functions $g, f:[0, T] \times \mathscr{B}_{\mathrm{h}} \times \mathrm{X} \rightarrow \mathrm{X}, \sigma:[0, \mathrm{~T}] \rightarrow \mathcal{L}_{2}^{0}(\mathrm{Y}, \mathrm{X})$ are respectively defined by

$$
g(t, x(t-k, \zeta)) d s=\bar{g}(t, x(t-k, \zeta)) d s),
$$




$$
f(t, x(t-k, \zeta)) d s)+c(\zeta) u(t)=\bar{f}(t, x(t-k, \zeta)) d s)+c(\zeta) u(t)
$$

The above system (4.1) can be written in the abstract from (1.1). Further, we assume that the following conditions hold.

$B: U \rightarrow X$ is a bounded linear operator defined by

$$
\mathrm{Bu}(\mathrm{t})(\zeta)=\mathrm{c}(\zeta) \mathrm{u}(\mathrm{t}), \quad 0 \leqslant \zeta \leqslant \pi, \quad \mathrm{u} \in \mathcal{L}^{2}([0, \mathrm{~T}] ; \mathrm{U}),
$$

and the linear operator $\mathrm{W}: \mathcal{L}^{2}([0, \mathrm{~T}] ; \mathrm{U}) \rightarrow \mathrm{X}$ is given by

$$
W u(\zeta)=\int_{0}^{T} R(T-s) c(\zeta) u(t) d s, \quad 0 \leqslant \zeta \leqslant \pi
$$

$W$ is a bounded linear operator but not necessarily one-to-one.

Let $\operatorname{ker} W=\left\{x \in \mathcal{L}^{2}([0, T] ; U), W x=0\right\}$ be the null space of $W$ and $[\text { ker } W]^{\perp}$ be its orthogonal complement in $\mathcal{L}^{2}([0, T] ; U)$. Let $\widetilde{W}=[\operatorname{ker} W]^{\perp} \rightarrow \operatorname{Range}(W)$ be the restriction of $W$ to $[\operatorname{ker} W]^{\perp}, W$ is necessarily one-to-one operator. The inverse mapping theorem says that $\widetilde{W}^{-1}$ is bounded since $[\text { ker } W]^{\perp}$ and Range $(W)$ are Banach spaces. So that $W^{-1}$ is bounded and takes values in $\mathcal{L}^{2}([0, T] ; U) \operatorname{ker} W$, hypothesis (H8) is satisfied. Thus, it is possible to verify that the assumptions on Theorem 3.2 are fulfilled and hence, the system (4.1) is controllable on $(-\infty, T]$.

\section{Conclusion}

In this paper we have proved the controllability results of neutral impulsive stochastic functional integrodifferential equations driven by a fractional Brownian motion with infinite delay in a real separable Hilbert space. We have used the techniques of stochastic analysis, the theory of resolvent operator in the sense of Grimmer, and Krasnoselskii fixed point theorem to study the controllability results with the illustrative example to justify the theory. One can extend the same problem for second order neutral impulsive stochastic functional integrodifferential equations with infinite delay using sine and cosine operators. Also, we can study the fractional order neutral impulsive stochastic functional integrodifferential system with infinite delay using Riemann-Liouville and Caputo derivatives as future work.

\section{Acknowledgment}

Authors are thankful to the anonymous referees for careful consideration and valuable comments.

\section{References}

[1] A. Anguraj, K. Ramkumar, Approximate controllability of semilinear stochastic integrodifferential system with nonlocal conditions, Fractal Fract., 2 (2018), 13 pages. 1

[2] G. Arthi, J. H. Park, H. Y. Jung, Existence and exponential stability for neutral stochastic integrodifferential equations with impulses driven by a fractional Brownian motion, Commun. Nonlinear Sci. Numer. Simul., 32 (2016), 145-157. 1

[3] K. Balachandran, S. Karthikeyan, J. H. Kim, Controllability of semilinear stochastic integrodifferential equations, Kybernetika, 43 (2007), 31-44.

[4] B. Boufoussi, S. Hajji, Neutral stochastic functional differential equations driven by a fractional Brownian motion in a Hilbert space, Statist. Probab. Lett., 82 (2012), 1549-1558. 1

[5] B. Boufoussi, S. Hajji, E. Lakhel, Functional differential equations in Hilbert spaces driven by a fractional Brownian motion, Afr. Mat., 23 (2012), 173-194. 1

[6] M. Chen, Approximate controllability of stochastic equations in a Hilbert space with fractional Brownian motions, Stoch. Dyn., 15 (2015), 16 pages. 1

[7] M. A. Diop, R. Sakthivel, A. A. Ndiaye, Neutral stochastic integrodifferential equations driven by a fractional Brownian motion with impulsive effects and time varying delays, Mediterr. J. Math., 13 (2016), 2425-2442. 1, 2 
[8] R. C. Grimmer, Resolvent operators for integral equations in a Banach space, Trans. Amer. Math. Soc., 273 (1982), 333-349. 2.1, 2.3, 2.1, 2.4, 2.7

[9] J. Klamka, Stochastic controllability of linear systems with delay in control, Bull. Pol. Acad. Sci. Tech. Sci., 55 (2007), 23-29. 1

[10] J. Klamka, Controllability of dynamical systems-A survey, Bull. Pol. Acad. Sci. Tech. Sci., 61 (2013), 221-229. 1

[11] E. Lakhel, Controllability of neutral stochastic functional integrodifferential equations driven by fractional Brownian motion, Stoch. Anal. Appl., 34 (2016), 427-440. 1

[12] E. Lakhel, S. Hajji, Existence and Uniqueness of Mild Solutions to NFSDEs Driven by a Fractional Brownian Motion With Non-Lipschitz Coefficients, J. Numer. Math. Stoch., 7 (2015), 14-29. 1

[13] Y. Li, B. Liu, Existence of solution of nonlinear neutral functional differential inclusion with infinite delay, Stoch. Anal. Appl., 25 (2007), 397-415. 2, 2.1, 2.2

[14] J. Liang, J. H. Liu, T.-J. Xiao, Nonlocal problems for integrodifferential equations, Dyn. Contin. Discrete Impuls. Syst. Ser. A Math. Anal., 15 (2008), 815-824. 2.5

[15] J. Y. Park, K. Balachandran, G. Arthi, Controllability of impulsive neutral integrodifferential systems with infinite delay in Banach spaces, Nonlinear Anal. Hybrid Syst., 3 (2009), 184-194. 1

[16] A. Pazy, Semigroups of Linear Operators and Applications to Partial Differential Equations, Springer-Verlag, New York, (1983).

[17] Y. Ren, X. Cheng, R. Sakthivel, On time dependent stochastic evolution equations driven by fractional Brownian motion in Hilbert space with finite delay, Math. Methods Appl. Sci., 37 (2014), 2177-2184. 1

[18] R. Sakthivel, R. Ganesh, Y. Ren, S. M. Anthoni, Approximate controllability of nonlinear fractional dynamical systems, Commun. Nonlinear Sci. Numer. Simul., 18 (2013), 3498-3508. 1, 2

[19] R. Sakthivel, J. Luo, Asymptotic stability of nonlinear impulsive stochastic differential equations, Statist. Probab. Lett., 79 (2009), 1219-1223. 